\title{
Fonctions maximales centrées de Hardy-Littlewood sur les groupes de Heisenberg
}

\author{
par \\ Hong-QUAN Li (Shanghai)
}

\begin{abstract}
By getting uniformly asymptotic estimates for the Poisson kernel on Heisenberg groups $\mathbb{H}_{2 n+1}$, we prove that there exists a constant $A>0$, independent of $n \in \mathbb{N}^{*}$, such that for all $f \in L^{1}\left(\mathbb{H}_{2 n+1}\right)$, we have $\|M f\|_{L^{1, \infty}} \leq A n\|f\|_{1}$, where $M$ denotes the centered Hardy-Littlewood maximal function defined by the Carnot-Carathéodory distance or by the Korányi norm.
\end{abstract}

1. Introduction. Sur $\mathbb{R}^{n}\left(n \in \mathbb{N}^{*}\right)$, notons $M$ la fonction maximale standard de Hardy-Littlewood, c'est-à-dire

$$
M f(x)=\sup _{r>0} \frac{1}{|B(x, r)|} \int_{B(x, r)}|f(y)| d y, \quad x \in \mathbb{R}^{n}, f \in L_{\text {loc }}^{1}\left(\mathbb{R}^{n}\right) .
$$

Dans [8], Stein et Strömberg ont obtenu le résultat suivant :

ThÉORÈme 1.1 (Stein-Strömberg). Il existe une constante $A>0$, indépendante de $n$, telle que

$$
|\{x ; M f(x)>\lambda\}| \leq A n \lambda^{-1}\|f\|_{1}, \quad \forall \lambda>0, f \in L^{1}\left(\mathbb{R}^{n}\right) .
$$

On rappelle qu'une estimation de type

$$
\|M f\|_{p} \leq A_{p}\|f\|_{p}, \quad \forall f \in L^{p}(1<p \leq+\infty),
$$

(où la constante ne dépend pas de $n$ ) a aussi été obtenue dans [8].

Le but de cette note est d'obtenir une estimation de type (1.1) dans le cadre des groupes de Heisenberg. Avant de donner notre résultat, nous rappelons quelques définitions et notations.

Rappelons (voir par exemple [4]) que le groupe de Heisenberg de dimension réelle $2 n+1\left(n \in \mathbb{N}^{*}\right), \mathbb{H}_{2 n+1}=\mathbb{C}^{n} \times \mathbb{R}$, est un groupe de Lie stratifié pour la loi $(z, t) \cdot\left(z^{\prime}, t^{\prime}\right)=\left(z+z^{\prime}, t+t^{\prime}+2 \Im\left\langle z, z^{\prime}\right\rangle\right)$, avec $z=\left(z_{1}, \ldots, z_{n}\right)$, $z^{\prime}=\left(z_{1}^{\prime}, \ldots, z_{n}^{\prime}\right) \in \mathbb{C}^{n}, z_{j}=x_{j}+\imath y_{j}\left(x_{j}, y_{j} \in \mathbb{R}\right),\left\langle z, z^{\prime}\right\rangle=\sum_{j=1}^{n} z_{j} \cdot \overline{z_{j}^{\prime}}$.

2000 Mathematics Subject Classification: 42B25, 43A80.

Key words and phrases: centered Hardy-Littlewood maximal function, Heisenberg groups, Poisson kernel, Carnot-Carathéodory distance. 
Dans la suite, on note $o=(0,0)$ l'origine de $\mathbb{H}_{2 n+1} ; g=(z, t) \in \mathbb{C}^{n} \times \mathbb{R}$ désignera un point de $\mathbb{H}_{2 n+1}$; et on note $\|z\|^{2}=\sum_{j=1}^{n}\left\|z_{j}\right\|^{2}$. On rappelle que la mesure de Haar sur $\mathbb{H}_{2 n+1}, d g$, est celle de Lebesgue.

Le sous-Laplacien sur $\mathbb{H}_{2 n+1}$ s'écrit $\Delta=\sum_{j=1}^{n}\left(\mathrm{X}_{j}^{2}+\mathrm{Y}_{j}^{2}\right)$, où $\mathrm{X}_{j}$ et $\mathrm{Y}_{j}$ $(1 \leq j \leq n)$ sont les champs de vecteurs, invariants à gauche sur $\mathbb{H}_{2 n+1}$, définis par

$$
\mathrm{X}_{j}=\frac{\partial}{\partial x_{j}}+2 y_{j} \frac{\partial}{\partial t}, \quad \mathrm{Y}_{j}=\frac{\partial}{\partial y_{j}}-2 x_{j} \frac{\partial}{\partial t} .
$$

Il y a deux distances standard sur $\mathbb{H}_{2 n+1}$ : distance de Carnot-Carathéodory $d_{\mathrm{CC}}$ (associée à $\left\{\mathrm{X}_{1}, \ldots, \mathrm{X}_{n}, \mathrm{Y}_{1}, \ldots, \mathrm{Y}_{n}\right\}$, voir par exemple [9]) et celle définie par la norme de Korányi $d_{\mathrm{K}}$ liée à la solution fondamentale de $\Delta$ (c'est-à-dire la fonction de Green, voir par exemple [3]). De plus, on a $d_{\mathrm{CC}}\left(g g_{1}, g g_{2}\right)=d_{\mathrm{CC}}\left(g_{1}, g_{2}\right), \quad d_{\mathrm{K}}\left(g g_{1}, g g_{2}\right)=d_{\mathrm{K}}\left(g_{1}, g_{2}\right), \quad \forall g, g_{1}, g_{2} \in \mathbb{H}_{2 n+1}$.

Par convention, on note $d_{\mathrm{K}}(g)=d_{\mathrm{K}}(g, o)$ et $d_{\mathrm{CC}}(g)=d_{\mathrm{CC}}(g, o)$. Rappelons que (voir par exemple [3])

$$
d_{\mathrm{K}}(z, t)=\left(\|z\|^{4}+t^{2}\right)^{1 / 4}, \quad \forall(z, t) \in \mathbb{H}_{2 n+1}=\mathbb{C}^{n} \times \mathbb{R} .
$$

Notons $\left.\mu(\varphi)=(2 \varphi-\sin 2 \varphi) /\left(2 \sin ^{2} \varphi\right):\right]-\pi, \pi\left[\rightarrow \mathbb{R}\right.$ et $\mu^{-1}$ sa fonction réciproque. On a $d_{\mathrm{CC}}^{2}(0, t)=\pi|t|$ et pour $z \neq 0(\operatorname{voir}[1])$

$$
d_{\mathrm{CC}}^{2}(z, t)=(\theta / \sin \theta)^{2}\|z\|^{2} \quad \text { avec } \theta=\mu^{-1}\left(t /\|z\|^{2}\right) .
$$

Dans la suite, on note $B_{\mathrm{K}}(g, r)\left(g \in \mathbb{H}_{2 n+1}, r>0\right)$ la boule ouverte de centre $g$ et de rayon $r$ définie par la norme de Korányi, et $B_{\mathrm{CC}}(g, r)$ celle définie par la distance de Carnot-Carathéodory. Si $E$ est un ensemble mesurable, alors on note $|E|$ son volume et $\chi_{E}$ sa fonction caractéristique. Pour $f \in L_{\text {loc }}^{1}\left(\mathbb{H}_{2 n+1}\right)$, on définit les deux fonctions maximales centrées de Hardy-Littlewood $M_{\mathrm{K}} f$ et $M_{\mathrm{CC}} f$ respectivement par

$$
\begin{array}{cc}
M_{\mathrm{K}} f(g)=\sup _{r>0}\left|B_{\mathrm{K}}(g, r)\right|^{-1} \int_{B_{\mathrm{K}}(g, r)}\left|f\left(g^{\prime}\right)\right| d g^{\prime}, & \forall g \in \mathbb{H}_{2 n+1}, \\
M_{\mathrm{CC}} f(g)=\sup _{r>0}\left|B_{\mathrm{CC}}(g, r)\right|^{-1} \int_{B_{\mathrm{CC}}(g, r)}\left|f\left(g^{\prime}\right)\right| d g^{\prime}, \quad \forall g \in \mathbb{H}_{2 n+1} .
\end{array}
$$

On a alors

ThÉORÈME 1.2. Il existe une constante $L>0$ telle que pour tout $n \in \mathbb{N}^{*}$,

$$
\|M f\|_{L^{1, \infty}} \leq L n\|f\|_{1}, \quad \forall f \in L^{1}\left(\mathbb{H}_{2 n+1}\right),
$$

avec $M=M_{\mathrm{K}}$ ou bien $M=M_{\mathrm{CC}}$.

On rappelle qu'une estimation de type (1.2) a été obtenue par J. Zienkiewicz [10].

L'idée principale de la démonstration du théorème 1.2 est d'utiliser "the Hopf-Dunford-Schwartz maximal ergodic theorem" (voir [2, pp. 690-691]) 
comme dans [8]. Cependant, on utilisera le noyau de Poisson au lieu du noyau de la chaleur utilisé dans [8].

Cette note est organisée de la façon suivante : Pour mieux comprendre notre méthode, on donnera dans la section 2 une nouvelle démonstration du théorème 1.1 en utilisant la formule explicite du noyau de Poisson sur $\mathbb{R}^{n}$. On étudiera les estimations uniformément asymptotiques du noyau de Poisson sur $\mathbb{H}_{2 n+1}$ dans la section 3 . La démonstration du théorème 1.2 sera donnée dans la section 4 pour $M=M_{\mathrm{K}}$ et dans la section 5 pour $M=M_{\mathrm{CC}}$.

Notations. Pour deux fonctions $f$ et $g$, on dit que $f=O(g)$ s'il existe une constante $c>0$ telle que $|f| \leq c|g|$; que $f=o(g)$ si $\lim f / g=0$; et que $f \sim g$ s'il existe une constante $A>1$ telle que $A^{-1} f \leq g \leq A f$.

2. Une nouvelle démonstration du théorème 1.1. Afin de mieux comprendre l'idée de la démonstration pour le cas des groupes de Heisenberg, on va montrer le théorème 1.1 en utilisant le noyau de Poisson.

Considérons le semi-groupe de Poisson sur $\mathbb{R}^{n}, e^{-t \sqrt{-\Delta}} f=f * P_{t}(t>0)$, où

$$
P_{t}(x)=\left[\frac{n}{2}|B(o, 1)| B\left(\frac{n}{2}, \frac{1}{2}\right)\right]^{-1} \frac{t}{\left(t^{2}+|x|^{2}\right)^{(n+1) / 2}},
$$

$B(n / 2,1 / 2)$ étant la fonction de Beta d'indices $n / 2$ et $1 / 2$. Comme

$$
P_{t}(x) \geq 0, \quad \forall x \in \mathbb{R}^{n}, \quad\left\|P_{t}\right\|_{1}=1, \quad \forall t>0,
$$

par "the Hopf-Dunford-Schwartz maximal ergodic theorem", on a

$$
\left|\left\{x ; \sup _{s>0} \frac{1}{s} \int_{0}^{s} e^{-t \sqrt{-\Delta}} f(x) d t>\lambda\right\}\right| \leq \frac{2}{\lambda}\|f\|_{1}, \quad \forall \lambda>0, f \in L^{1}\left(\mathbb{R}^{n}\right) .
$$

Pour prouver le théorème 1.1, il nous reste à montrer qu'il existe une constante $A>0$ telle que pour tout $n \in \mathbb{N}^{*}$, on a

$$
M f(x) \leq A n \sup _{s>0} \frac{1}{s} \int_{0}^{s} e^{-t \sqrt{-\Delta}} f(x) d t, \quad \forall x \in \mathbb{R}^{n}, 0 \leq f \in L^{1}\left(\mathbb{R}^{n}\right) .
$$

Pour $g_{0} \in \mathbb{R}^{n}$, notons $L_{g_{0}}$ l'opérateur de translation à gauche défini par $g_{0}$, c'est-à-dire $L_{g_{0}} f(g)=f\left(g_{0}+g\right)$; on remarque que $L_{g_{0}} M=M L_{g_{0}}$ et que $L_{g_{0}} e^{-t \sqrt{-\Delta}}=e^{-t \sqrt{-\Delta}} L_{g_{0}}$. Par la structure de dilatation sur $\mathbb{R}^{n}$, il suffit de trouver un $s(n)>0$ tel que pour tout $x \neq o$, on a

$$
\begin{aligned}
|B(o, 1)|^{-1} \chi_{B(o, 1)}(x) & \leq A n \frac{1}{s(n)} \int_{0}^{s(n)} P_{t}(x) d t \\
= & {\left[\frac{n}{2}|B(o, 1)| B\left(\frac{n}{2}, \frac{1}{2}\right)\right]^{-1} \frac{A n}{s(n)} \int_{0}^{s(n)} \frac{t}{\left(t^{2}+|x|^{2}\right)^{(n+1) / 2}} d t . }
\end{aligned}
$$


Il nous reste à vérifier que pour un certain $A>0$ et un certain $s(n)>0$,

$$
\begin{aligned}
1 & \leq 2 A\left[B\left(\frac{n}{2}, \frac{1}{2}\right)\right]^{-1} \frac{1}{s(n)} \int_{0}^{s(n)} \frac{t}{\left(t^{2}+1\right)^{(n+1) / 2}} d t \\
& =2 A \frac{1}{B(n / 2,1 / 2)} \frac{1}{s(n)} \frac{1}{n-1}\left[1-e^{-\frac{n-1}{2} \ln \left(1+s^{2}(n)\right)}\right] .
\end{aligned}
$$

En distinguant les trois cas $s(n) \ll n^{-1 / 2}, s(n) \sim n^{-1 / 2}$ et $s(n) \gg n^{-1 / 2}$, par le développement de Taylor d'ordre 1 autour de l'origine pour $\ln (1+a)$ et $e^{a}(|a| \ll 1)$, on voit facilement que pour maximiser

$$
\frac{1}{s(n)}\left[1-e^{-\frac{n-1}{2} \ln \left(1+s^{2}(n)\right)}\right]
$$

on doit choisir $s(n) \sim n^{-1 / 2}$ et on aura

$$
\frac{1}{s(n)}\left[1-e^{-\frac{n-1}{2} \ln \left(1+s^{2}(n)\right)}\right] \sim n^{1 / 2} .
$$

Par le fait que $B(n / 2,1 / 2) \sim n^{-1 / 2}$, on peut choisir $A>0$ assez grand tel que (2.1) soit satisfaite.

3. Estimations uniformément asymptotiques du noyau de Poisson sur $\mathbb{H}_{2 n+1}$. Soient $p_{h}=p_{h}^{(n)}(h>0)$ le noyau de la chaleur (c'est-à-dire le noyau intégral de $e^{h \Delta}$ ) sur $\mathbb{H}_{2 n+1}$ et $P_{h}=P_{h}^{(n)}$ le noyau de Poisson (c'està-dire le noyau intégral de $\left.e^{-h \sqrt{-\Delta}}\right)$. Par convention, on note $p_{h}(g)=p_{h}(g, o)$ et $p(g)=p_{1}(g)$. De la même façon, on note $P_{h}(g)=P_{h}(g, o)$ et $P(g)=P_{1}(g)$. Par [5], [4] ou [6], on a l'expression explicite de $p_{h}$ comme suit :

$$
p_{h}(z, t)=\frac{1}{2(4 \pi h)^{n+1}} \int_{\mathbb{R}} \exp \left(\frac{\lambda}{4 h}\left(\imath t-\|z\|^{2} \operatorname{coth} \lambda\right)\right)\left(\frac{\lambda}{\sinh \lambda}\right)^{n} d \lambda .
$$

Notons $Q=2 n+2$ la dimension homogène de $\mathbb{H}_{2 n+1}$. Rappelons que

$$
P_{h}(z, t)=h^{-Q} P\left(z / h, t / h^{2}\right), \quad \forall h>0,(z, t) \in \mathbb{C}^{n} \times \mathbb{R} .
$$

On a les estimations uniformément asymptotiques de $P$, donc celles de $P_{h}$, comme suit :

Proposition 3.1. Soit $U \gg$ 1. Lorsque $n \rightarrow+\infty$, pour $d_{\mathrm{K}}(z, t) \geq U \sqrt{n}$, on $a$

$$
\begin{aligned}
P(z, t)= & \left(\frac{\sin \phi}{\phi}\right)^{3 / 2} \frac{\Gamma(n+3 / 2)}{2 \pi^{n+3 / 2}} B\left(\frac{n+5 / 2}{2}-\frac{1}{2}, \frac{1}{2}\right) d_{\mathrm{K}}^{-Q-1}(z, t) \\
& \times\left(1+O\left(\frac{n}{d_{\mathrm{K}}^{2}(z, t)}\right)\right)\left(1+O\left(n^{-1 / 2}\right)\right),
\end{aligned}
$$

où $0 \leq \phi \leq \pi / 2$ est déterminé par $e^{-\imath \phi}=d_{\mathrm{K}}^{-2}(z, t)\left(\|z\|^{2}-\imath|t|\right)$. 
Preuve. Rappelons que

$$
e^{-\sqrt{-\Delta}}=\frac{1}{2 \sqrt{\pi}} \int_{0}^{+\infty} h^{-3 / 2} e^{-1 /(4 h)} e^{h \Delta} d h ;
$$

on insère (3.1) dans la formule précédente, et par le théorème de Fubini, on obtient

$$
\begin{aligned}
& P(z, t)=\frac{1}{2 \sqrt{\pi}} \frac{1}{2(4 \pi)^{n+1}} \\
& \times \int_{\mathbb{R}}\left[\int_{0}^{+\infty} h^{-n-5 / 2} \exp \left(\frac{1}{4 h}\left(\imath t \lambda-\|z\|^{2} \lambda \operatorname{coth} \lambda-1\right)\right) d h\right]\left(\frac{\lambda}{\sinh \lambda}\right)^{n} d \lambda .
\end{aligned}
$$

Par le changement de variable

$$
\gamma=\frac{1+\|z\|^{2} \lambda \operatorname{coth} \lambda-\imath t \lambda}{4 h},
$$

on voit que

$$
\begin{aligned}
\int_{0}^{+\infty} h^{-n-5 / 2} \exp ( & \left.\frac{1}{4 h}\left(\imath t \lambda-\|z\|^{2} \lambda \operatorname{coth} \lambda-1\right)\right) d h \\
= & {\left[\frac{1+\|z\|^{2} \lambda \operatorname{coth} \lambda-\imath t \lambda}{4}\right]^{-n-3 / 2} \int_{0}^{+\infty} \gamma^{n+1 / 2} e^{-\gamma} d \gamma } \\
& =\left[\frac{4}{1+\|z\|^{2} \lambda \operatorname{coth} \lambda-\imath t \lambda}\right]^{n+3 / 2} \Gamma\left(n+\frac{3}{2}\right),
\end{aligned}
$$

donc

$$
\begin{aligned}
P(z, t) & =\frac{\Gamma(n+3 / 2)}{2 \pi^{n+3 / 2}} \int_{\mathbb{R}}\left(\frac{\lambda}{\sinh \lambda}\right)^{n}\left(1+\|z\|^{2} \lambda \operatorname{coth} \lambda-\imath t \lambda\right)^{-n-3 / 2} d \lambda \\
& =\frac{\Gamma(n+3 / 2)}{2 \pi^{n+3 / 2}} \int_{\mathbb{R}}\left(\frac{\lambda}{\sinh \lambda}\right)^{n}\left(1+\|z\|^{2} \lambda \operatorname{coth} \lambda-\imath|t| \lambda\right)^{-n-3 / 2} d \lambda .
\end{aligned}
$$

Posons

$$
f(\|z\|,|t|, \lambda)=\|z\|^{2} \lambda \operatorname{coth} \lambda-\imath|t| \lambda .
$$

On sait bien que $\Re f(\|z\|,|t|, \lambda) \geq 0$ pour tout $0 \leq \Im \lambda \leq \pi / 2$. En fait, pour tout $0 \leq \theta \leq \pi / 2$ et tout $\iota \in \mathbb{R}$, on a (voir [1, p. 645])

$$
\Re f(\|z\|,|t|, \imath \theta)=\frac{\theta}{\sin \theta} \cos \theta\|z\|^{2}+|t| \theta \geq 0,
$$


et

$$
\begin{aligned}
& \Re\{f(\|z\|,|t|, \iota+\imath \theta)-f(\|z\|,|t|, \imath \theta)\} \\
& \quad=\|z\|^{2} \frac{\sinh ^{2} \iota}{\sinh ^{2} \iota+\sin ^{2} \theta}(\iota \operatorname{coth} \iota-\theta \cot \theta) \geq 0 .
\end{aligned}
$$

Notons

$$
F(\lambda)=\left(\frac{\lambda}{\sinh \lambda}\right)^{n}\left(1+\|z\|^{2} \lambda \operatorname{coth} \lambda-\imath|t| \lambda\right)^{-n-3 / 2} .
$$

Alors on voit facilement que $F$ est analytique sur

$$
\Omega=\{\lambda \in \mathbb{C} ; 0<\Im \lambda<\pi / 2\},
$$

et continue sur $\bar{\Omega}$. De plus,

$$
\lim _{\lambda \in \bar{\Omega},|\lambda| \rightarrow+\infty}|F(\lambda)|=0 .
$$

Par le théorème fondamental de Cauchy, on a donc

$$
\int_{\mathbb{R}} F(\lambda) d \lambda=\int_{\mathbb{R}} F(\lambda+\imath \phi) d \lambda=W
$$

avec

$$
0 \leq \phi \leq \pi / 2, \quad e^{-\imath \phi}=d_{\mathrm{K}}^{-2}(z, t)\left(\|z\|^{2}-\imath|t|\right)
$$

Or,

$$
\begin{aligned}
f(\|z\|,|t|, \lambda) & =\frac{\lambda}{\sinh \lambda}\left(\|z\|^{2} \cosh \lambda-\imath|t| \sinh \lambda\right) \\
& =\frac{\lambda}{\sinh \lambda} d_{\mathrm{K}}^{2}(z, t)(\cosh \lambda \cos \phi-\imath \sinh \lambda \sin \phi) \\
& =\frac{\lambda}{\sinh \lambda} d_{\mathrm{K}}^{2}(z, t) \cosh (\lambda-\imath \phi),
\end{aligned}
$$

et donc

$$
\begin{aligned}
W & =\int_{\mathbb{R}}\left(\frac{\lambda+\imath \phi}{\sinh (\lambda+\imath \phi)}\right)^{n}\left(1+\frac{\lambda+\imath \phi}{\sinh (\lambda+\imath \phi)} d_{\mathrm{K}}^{2}(z, t) \cosh \lambda\right)^{-n-3 / 2} d \lambda \\
& =\int_{\mathbb{R}}\left(\frac{\sinh (\lambda+\imath \phi)}{\lambda+\imath \phi}\right)^{3 / 2}\left(\frac{\sinh (\lambda+\imath \phi)}{\lambda+\imath \phi}+d_{\mathrm{K}}^{2}(z, t) \cosh \lambda\right)^{-n-3 / 2} d \lambda .
\end{aligned}
$$

Par conséquent, pour terminer la preuve de la proposition 3.1, il nous reste à montrer

LEMme 3.2. Soit $U \gg 1$. Lorsque $n \rightarrow+\infty$, pour $d_{\mathrm{K}}(z, t) \geq U \sqrt{n}$, on a

$$
\begin{aligned}
W= & \left(\frac{\sin \phi}{\phi}\right)^{3 / 2} B\left(\frac{n+5 / 2}{2}-\frac{1}{2}, \frac{1}{2}\right) \\
& \times d_{\mathrm{K}}^{-Q-1}(z, t)\left(1+O\left(n^{-1 / 2}\right)\right)\left(1+O\left(\frac{n}{d_{\mathrm{K}}^{2}(z, t)}\right)\right) .
\end{aligned}
$$


Preuve. Posons

$$
\begin{aligned}
& W_{1}=\int_{-1}^{1}\left(\frac{\sinh (\lambda+\imath \phi)}{\lambda+\imath \phi}\right)^{3 / 2}\left(\frac{\sinh (\lambda+\imath \phi)}{\lambda+\imath \phi}+d_{\mathrm{K}}^{2}(z, t) \cosh \lambda\right)^{-n-3 / 2} d \lambda, \\
& W_{2}=\int_{|\lambda| \geq 1}\left(\frac{\sinh (\lambda+\imath \phi)}{\lambda+\imath \phi}\right)^{3 / 2}\left(\frac{\sinh (\lambda+\imath \phi)}{\lambda+\imath \phi}+d_{\mathrm{K}}^{2}(z, t) \cosh \lambda\right)^{-n-3 / 2} d \lambda .
\end{aligned}
$$

On a alors $W=W_{1}+W_{2}$. On commence par estimer $W_{2}$. On constate d'abord que

$$
\left|\frac{\sinh (\lambda+\imath \phi)}{\lambda+\imath \phi}\right| \leq \cosh \lambda, \quad \forall 0 \leq \phi \leq \pi / 2,|\lambda| \geq 1 .
$$

Donc, pour $n \rightarrow+\infty$ avec $d_{\mathrm{K}}(z, t) \gg n^{1 / 2}$, on a

$$
\begin{aligned}
\left|W_{2}\right| & \leq \int_{|\lambda| \geq 1}\left[\left(d_{\mathrm{K}}^{2}(z, t)-1\right) \cosh \lambda\right]^{-n-3 / 2}(\cosh \lambda)^{3 / 2} d \lambda \\
& \leq 2\left(d_{\mathrm{K}}^{2}(z, t)-1\right)^{-n-3 / 2} \int_{1}^{+\infty}(\cosh \lambda)^{-n} \sinh \lambda d \lambda \\
& =2\left(d_{\mathrm{K}}^{2}(z, t)-1\right)^{-n-3 / 2} \frac{1}{n-1}(\cosh 1)^{1-n} \\
& =d_{\mathrm{K}}^{-Q-1}(z, t) e^{-(n+3 / 2) \ln \left(1-d_{\mathrm{K}}^{-2}(z, t)\right)} o\left(n^{-2}\right) \\
& =d_{\mathrm{K}}^{-Q-1}(z, t)\left(1+O\left(\frac{n}{d_{\mathrm{K}}^{2}(z, t)}\right)\right) o\left(n^{-2}\right) .
\end{aligned}
$$

Il nous reste à étudier $W_{1}$. On constate d'abord que pour $-1 \leq \lambda \leq 1$ et $0 \leq \phi \leq \pi / 2$, on a

$$
\left(\frac{\sinh (\lambda+\imath \phi)}{\lambda+\imath \phi}\right)^{3 / 2}=\left(\frac{\sin \phi}{\phi}\right)^{3 / 2}[1+O(\lambda)],
$$

et si de plus $n / d_{\mathrm{K}}^{2}(z, t) \ll 1$, alors

$$
\begin{aligned}
& \left(\frac{\sinh (\lambda+\imath \phi)}{\lambda+\imath \phi}+d_{\mathrm{K}}^{2}(z, t) \cosh \lambda\right)^{-n-3 / 2} \\
& =\left(d_{\mathrm{K}}^{2}(z, t) \cosh \lambda\right)^{-n-3 / 2} \\
& \quad \quad \times \exp \left(-\left(n+\frac{3}{2}\right) \ln \left(1+\frac{1}{d_{\mathrm{K}}^{2}(z, t) \cosh \lambda} \frac{\sinh (\lambda+\imath \phi)}{\lambda+\imath \phi}\right)\right) \\
& =d_{\mathrm{K}}^{-Q-1}(z, t)(\cosh \lambda)^{-n-3 / 2}\left[1+O\left(\frac{n}{d_{\mathrm{K}}^{2}(z, t)}\right)\right] .
\end{aligned}
$$


On peut donc écrire

$$
\begin{aligned}
W_{1}= & \left(\frac{\sin \phi}{\phi}\right)^{3 / 2} d_{\mathrm{K}}^{-Q-1}(z, t) \\
& \times \int_{-1}^{1}(\cosh \lambda)^{-n-3 / 2}\left[1+O\left(\frac{n}{d_{\mathrm{K}}^{2}(z, t)}\right)\right][1+O(\lambda)] d \lambda .
\end{aligned}
$$

Par conséquent, pour terminer la preuve du lemme 3.2, il faut montrer que

$$
\begin{aligned}
& \int_{-1}^{1}(\cosh \lambda)^{-n-3 / 2}\left[1+O\left(\frac{n}{d_{\mathrm{K}}^{2}(z, t)}\right)\right][1+O(\lambda)] d \lambda \\
& \quad=B\left(\frac{n+5 / 2}{2}-\frac{1}{2}, \frac{1}{2}\right)\left(1+O\left(n^{-1 / 2}\right)\right)\left(1+O\left(\frac{n}{d_{\mathrm{K}}^{2}(z, t)}\right)\right) .
\end{aligned}
$$

En fait, d'une part, on remarque d'abord que

$$
\int_{-1}^{1}(\cosh \lambda)^{-n-3 / 2} d \lambda=2\left[\int_{0}^{+\infty}-\int_{1}^{+\infty}\right](\cosh \lambda)^{-n-3 / 2} d \lambda
$$

puis, par le fait que

$$
\begin{aligned}
\int_{0}^{+\infty}(\cosh \lambda)^{-n-3 / 2} d \lambda & =\int_{0}^{+\infty}(\cosh \lambda)^{-n-5 / 2} d(\sinh \lambda) \\
& =\frac{1}{2} B\left(\frac{n+5 / 2}{2}-\frac{1}{2}, \frac{1}{2}\right) \quad(\text { voir }[7, \text { pp. 6-7]) } \\
\int_{1}^{+\infty}(\cosh \lambda)^{-n-3 / 2} d \lambda & \leq \int_{1}^{+\infty}(\cosh \lambda)^{-n-3 / 2} \sinh \lambda d \lambda \\
& =\frac{1}{n+1 / 2}(\cosh 1)^{-n-1 / 2}
\end{aligned}
$$

et que

$$
\begin{aligned}
B\left(\frac{n+5 / 2}{2}-\frac{1}{2}, \frac{1}{2}\right) & =\Gamma(1 / 2) \frac{\Gamma((n+5 / 2) / 2-1 / 2)}{\Gamma((n+5 / 2) / 2)} \\
& =\sqrt{\pi}\left(\frac{n+5 / 2}{2}\right)^{-1 / 2}(1+o(1)) \quad \text { lorsque } n \rightarrow+\infty
\end{aligned}
$$

(voir $[7$, p. 6 et p. 12]), on a

$$
\int_{-1}^{1}(\cosh \lambda)^{-n-3 / 2} d \lambda=B\left(\frac{n+5 / 2}{2}-\frac{1}{2}, \frac{1}{2}\right)\left[1+O\left(n^{-1 / 2}\right)\right], \quad n \rightarrow+\infty .
$$


D'autre part,

$$
\begin{aligned}
\int_{-1}^{1}|\lambda|(\cosh \lambda)^{-n-3 / 2} d \lambda & =2 \int_{0}^{1} \lambda(\cosh \lambda)^{-n-3 / 2} d \lambda \\
& \leq 2 \int_{0}^{+\infty}(\cosh \lambda)^{-n-3 / 2} \sinh \lambda d \lambda \\
& =\frac{2}{n+1 / 2} .
\end{aligned}
$$

On obtient donc (3.2).

4. Preuve du théorème $\mathbf{1 . 2}$ pour $M=M_{\mathrm{K}}$. En répétant la méthode utilisée dans la section 2 , il suffit de montrer qu'il existe une constante $L>0$ telle que pour tout $n \in \mathbb{N}^{*}$, avec un $s(n)>0$ bien choisi, on a pour tout $(z, t) \neq o$,

$$
\begin{aligned}
\left|B_{\mathrm{K}}(o, 1)\right|^{-1} \chi_{B_{\mathrm{K}}(o, 1)}(z, t) & \leq \operatorname{Ln} \frac{1}{s(n)} \int_{0}^{s(n)} P_{h}(z, t) d h \\
& =\operatorname{Ln} \frac{1}{s(n)} \int_{0}^{s(n)} h^{-Q} P\left(\frac{z}{h}, \frac{t}{h^{2}}\right) d h .
\end{aligned}
$$

Choisissons $U \gg 1$ et $s(n)=(U \sqrt{n})^{-1}$. Pour $0<d_{\mathrm{K}}(z, t)<1$, on constate d'abord que

$$
\frac{1}{s(n)} \int_{0}^{s(n)} h^{-Q} P\left(\frac{z}{h}, \frac{t}{h^{2}}\right) d h \geq U \sqrt{n} \int_{0}^{d_{\mathrm{K}}(z, t) /(U \sqrt{n})} h^{-Q} P\left(\frac{z}{h}, \frac{t}{h^{2}}\right) d h,
$$

puis, par la proposition 3.1, que

$$
\begin{aligned}
& \frac{1}{s(n)} \int_{0}^{s(n)} h^{-Q} P\left(\frac{z}{h}, \frac{t}{h^{2}}\right) d h \\
& \quad \geq R(n ; z, t) U \sqrt{n} \int_{0}^{d_{\mathrm{K}}(z, t) / U \sqrt{n}} h d h\left(1+O\left(1 / U^{2}\right)\right)\left(1+O\left(n^{-1 / 2}\right)\right),
\end{aligned}
$$

avec

$$
R(n ; z, t)=\left(\frac{\sin \phi}{\phi}\right)^{3 / 2} \frac{\Gamma(n+3 / 2)}{2 \pi^{n+3 / 2}} B\left(\frac{n+5 / 2}{2}-\frac{1}{2}, \frac{1}{2}\right) d_{\mathrm{K}}^{-Q-1}(z, t) .
$$

Si $U$ est choisi assez grand, lorsque $n \rightarrow+\infty$ on aura, pour tout $0<$ $d_{\mathrm{K}}(z, t)<1$ 


$$
\begin{aligned}
\frac{1}{s(n)} \int_{0}^{s(n)} h^{-Q} P & \left(\frac{z}{h}, \frac{t}{h^{2}}\right) d h \\
& \geq\left(\frac{\sin \phi}{\phi}\right)^{3 / 2} \frac{\Gamma(n+3 / 2)}{2 \pi^{n+3 / 2}} B\left(\frac{n+5 / 2}{2}-\frac{1}{2}, \frac{1}{2}\right) \frac{1}{4 U \sqrt{n}} .
\end{aligned}
$$

Puisque $\frac{\sin \phi}{\phi} \geq \frac{2}{\pi}$ pour tout $0 \leq \phi \leq \pi / 2$ et

$$
B\left(\frac{n+5 / 2}{2}-\frac{1}{2}, \frac{1}{2}\right) \sim n^{-1 / 2}, \quad n \rightarrow+\infty,
$$

pour montrer (4.1), il nous reste à montrer qu'il existe une constante $C>0$ telle que

$$
\frac{\Gamma(n+3 / 2)}{\pi^{n+3 / 2}}\left|B_{\mathrm{K}}(o, 1)\right| \geq C, \quad \forall n \in \mathbb{N}^{*} .
$$

En fait, on voit d'abord que

$$
\left|B_{\mathrm{K}}(o, 1)\right|=\int_{(z, t) \in \mathbb{C}^{n} \times \mathbb{R},\|z\|^{4}+|t|^{2}<1} d z d t=2\left|\mathrm{~S}^{2 n-1}\right| \int_{0}^{1} r^{2 n-1} \sqrt{1-r^{4}} d r,
$$

où $\left|S^{2 n-1}\right|$ désigne la surface de la sphère unitaire dans $\mathbb{R}^{2 n}$, puis que (voir $[7$, p. 8])

$$
\left|B_{\mathrm{K}}(o, 1)\right|=2^{-1}\left|\mathrm{~S}^{2 n-1}\right| B\left(\frac{n}{2}, \frac{3}{2}\right) .
$$

Comme $\left|\mathrm{S}^{2 n-1}\right|=2 \pi^{n} / \Gamma(n)$ et

$$
B\left(\frac{n}{2}, \frac{3}{2}\right) \sim n^{-3 / 2}, \quad \frac{\Gamma(n+3 / 2)}{\Gamma(n)} \sim n^{3 / 2}, \quad n \rightarrow+\infty,
$$

on obtient donc (4.2).

5. Preuve du théorème 1.2 pour $M=M_{\mathrm{CC}}$. On voit facilement d'après la section 4 que pour montrer le théorème 1.2 pour $M=M_{\mathrm{CC}}$, il suffit d'établir les deux lemmes suivants :

LEMME 5.1. On a $d_{\mathrm{K}}(z, t) \leq d_{\mathrm{CC}}(z, t)$ pour tout $(z, t) \in \mathbb{H}_{2 n+1}$.

LEMME 5.2. Il existe une constante $c>0$ telle que

$$
\left|B_{\mathrm{CC}}(o, 1)\right| \geq c\left|B_{\mathrm{K}}(o, 1)\right|, \quad \forall n \in \mathbb{N}^{*} .
$$

Preuve $d u$ lemme 5.1. Puisque $d(g)=d\left(g^{-1}\right)$ avec $d=d_{\mathrm{K}}$ ou bien $d=d_{\mathrm{CC}}$, on peut supposer dans la suite $t \geq 0$.

Comme $d_{\mathrm{K}}^{2}(0, t)=|t| \leq \pi|t|=d_{\mathrm{CC}}^{2}(0, t)$, il nous reste à montrer que pour $t \geq 0$ et $z \neq 0$, on a

$$
d_{\mathrm{K}}^{4}(z, t)=\|z\|^{4}+|t|^{2} \leq\left(\frac{\theta}{\sin \theta}\right)^{4}\|z\|^{4},
$$


avec $0 \leq \theta<\pi$ satisfaisant

$$
\frac{2 \theta-\sin 2 \theta}{2 \sin ^{2} \theta}=\frac{t}{\|z\|^{2}} .
$$

Autrement dit, il faut montrer que

$$
1+\left(\frac{\theta-\sin \theta \cos \theta}{\sin ^{2} \theta}\right)^{2} \leq\left(\frac{\theta}{\sin \theta}\right)^{4}, \quad \forall 0 \leq \theta<\pi .
$$

Posons

$$
g(\theta)=\theta^{4}-\sin ^{4} \theta-(\theta-\sin \theta \cos \theta)^{2}, \quad 0 \leq \theta<\pi .
$$

Il nous reste à montrer que $g(\theta) \geq 0$ pour tout $0 \leq \theta<\pi$. En fait, on a $g(0)=0$ et

$g^{\prime}(\theta)=4 \theta^{3}-4 \sin ^{3} \theta \cos \theta-2(\theta-\sin \theta \cos \theta) 2 \sin ^{2} \theta=4 \theta\left(\theta^{2}-\sin ^{2} \theta\right) \geq 0$.

Ceci achève la preuve du lemme.

Remarque. De la même façon, on peut montrer que

$$
d_{\mathrm{CC}}(z, t) \leq \sqrt{\pi} d_{\mathrm{K}}(z, t), \quad \forall(z, t) \in \mathbb{H}_{2 n+1} .
$$

Preuve du lemme 5.2. Pour $z \neq 0$, rappelons que $d_{\mathrm{CC}}(z, t)=(\theta / \sin \theta)\|z\|$ avec $-\pi<\theta<\pi$ déterminé par

$$
\mu(\theta)=\frac{2 \theta-\sin 2 \theta}{2 \sin ^{2} \theta}=\frac{t}{\|z\|^{2}} .
$$

On a

$$
\begin{aligned}
\left|B_{\mathrm{CC}}(o, 1)\right| & \iint_{(\theta / \sin \theta)\|z\|<1,-\pi<\theta<\pi, t=\mu(\theta)\|z\|^{2}} d z d t \\
& =\int_{0<\|z\|<1} 2 \mu\left(\theta_{0}(\|z\|)\right)\|z\|^{2} d z,
\end{aligned}
$$

où $0 \leq \theta_{0}(\|z\|)<\pi$ est défini par

$$
\frac{\sin \theta_{0}(\|z\|)}{\theta_{0}(\|z\|)}=\|z\|
$$

Donc

$$
\left|B_{\mathrm{CC}}(o, 1)\right|=\int_{0<\|z\|<1} \frac{2 \theta_{o}(\|z\|)-\sin 2 \theta_{0}(\|z\|)}{\theta_{0}^{2}(\|z\|)} d z .
$$

Rappelons que

$$
\left|B_{\mathrm{K}}(o, 1)\right|=\int_{0<\|z\|<1} 2 \sqrt{1-\|z\|^{4}} d z .
$$

Il nous reste à montrer que

$$
\inf _{0 \leq \theta<\pi} \frac{2 \theta-\sin 2 \theta}{\theta^{2}}\left(1-\left(\frac{\sin \theta}{\theta}\right)^{4}\right)^{-1 / 2}>0,
$$


dont la preuve est facile en utilisant le développement de Taylor d'ordre 3 autour de l'origine pour $\sin \varphi$.

Remerciements. L'auteur est partiellement supporté par "Shanghai Pujiang Program (grant no. 07pj14009)" et "Fok Ying Tong Education Foundation (grant no. 111001)". Je tiens à remercier D. Zaffran pour m'avoir aidé à rédiger cette note. Je voudrais aussi remercier le référé pour de nombreuses suggestions.

\section{Références}

[1] R. Beals, B. Gaveau and P. C. Greiner, Hamilton-Jacobi theory and the heat kernel on Heisenberg groups, J. Math. Pures Appl. (9) 79 (2000), 633-689.

[2] N. Dunford and J. T. Schwartz, Linear Operators. I. General Theory, with the assistance of W. G. Bade and R. G. Bartle, Interscience, New York, 1958.

[3] G. B. Folland, A fundamental solution for a subelliptic operator, Bull. Amer. Math. Soc. 79 (1973), 373-376.

[4] B. Gaveau, Principe de moindre action, propagation de la chaleur et estimées souselliptiques sur certains groupes nilpotents, Acta Math. 139 (1977), 95-153.

[5] A. Hulanicki, The distribution of energy in the Brownian motion in the Gaussian field and analytic-hypoellipticity of certain subelliptic operators on the Heisenberg group, Studia Math. 56 (1976), 165-173.

[6] F. Lust-Piquard, A simple-minded computation of heat kernels on Heisenberg groups, Colloq. Math. 97 (2003), 233-249.

[7] W. Magnus, F. Oberhettinger and R. P. Soni, Formulas and Theorems for the Special Functions of Mathematical Physics, Springer, New York, 1966.

[8] E. M. Stein and J.-O. Strömberg, Behavior of maximal functions in $\mathbb{R}^{n}$ for large $n$, Ark. Mat. 21 (1983), 259-269.

[9] N. Th. Varopoulos, L. Saloff-Coste and T. Coulhon, Analysis and Geometry on Groups, Cambridge Tracts in Math. 100, Cambridge Univ. Press, Cambridge, 1992.

[10] J. Zienkiewicz, Estimates for the Hardy-Littlewood maximal function on the Heisenberg group, Colloq. Math. 103 (2005), 199-205.

School of Mathematical Sciences

Fudan University

220 Handan Road

Shanghai 200433

People's Republic of China

E-mail: hongquan_li@fudan.edu.cn

hong_quanli@yahoo.fr

Received April 18, 2008

Revised version July 23, 2008 